\title{
Polynomial-Time Subgraph Enumeration for Automated Instruction Set Extension
}

\author{
Paolo Bonzini and Laura Pozzi \\ Faculty of Informatics \\ University of Lugano (USI) \\ Switzerland \\ Email: paolo.bonzini@lu.unisi.ch, laura.pozzi@unisi.ch
}

\begin{abstract}
This paper proposes a novel algorithm that, given a data-flow graph and an input/output constraint, enumerates all convex subgraphs under the given constraint in polynomial time with respect to the size of the graph. These subgraphs have been shown to represent efficient Instruction Set Extensions for customizable processors. The search space for this problem is inherently polynomial but, to our knowledge, this is the first paper to prove this and to present a practical algorithm for this problem with polynomial complexity. Our algorithm is based on properties of convex subgraphs that link them to the concept of multiple-vertex dominators. We discuss several pruning techniques that, without sacrificing the optimality of the algorithm, make it practical for data-flow graphs of a thousands nodes or more.
\end{abstract}

\section{Introduction}

A common practice in designing system-on-chip processors is to define a basic customizable processor and to extend it with units specialized for particular applications. Since typical embedded systems already include several Application-Specific Integrated Circuits (ASICs), it is conceivable to develop differently customized versions of the processor for each product. Such processor extensions can increase performance in specific domains, without the cost of advanced RISC processors and the complexity of entirely customized instruction sets. Many manufacturers are proposing customizable processors, such as Tensilica Xtensa and ARC ARCtangent. Instead of ASICs, other manufacturers use reconfigurable fabrics as accelerators. On some Xilinx systems, for example, an FPGA and a hardwired PowerPC processor coexist on the same die and share the same bus.

Independently of the technology adopted, it is important to be able to automatically generate the best performing instruction set extensions for an application. An exact solu- tion to this problem is not feasible if we want to generate more than one instruction [12]. On the other hand, several algorithms exist to find the single best performing instruction set extension in a graph. Exact algorithms for this problem may be based on Integer Linear Programming [2] or pruned exploration of the subgraphs [12,3].

An important subproblem in optimal ISE identification is enumeration of the valid subgraphs. While algorithms like $[12,3]$ focus on an exponential search space (each node of a basic block can be either in or out of a subgraph), we focus attention on the subgraphs input and output nodes. This way, as it can be seen in detail later, we identify a problem space that is no longer exponential in the size of the graph.

Based on this observation, we propose a new practical algorithm to enumerate all subgraphs under a given microarchitectural constraint, whose complexity is polynomial in the size of the graph. The algorithm is based on the relationship between convex subgraphs and multiple-vertex dominators. Even though the complexity is still rather highup to $O\left(n^{7}\right)$ for commonly used input/output constraintsseveral pruning techniques allow it to process basic blocks of around a thousand nodes in about a minute.

The next section discusses related work in the domain of instruction-set selection and overviews previous uses of multiple-vertex dominators. Section 3 provides a theoretical framework for the problem, and section 4 discusses the relationship between subgraph enumeration and multiplevertex dominators. Section 5 introduces the algorithm, together with some techniques to prune the search space. Finally, section 6 presents our experimental setup and discusses the results.

\section{Related work}

Past literature on custom instruction identification can be roughly divided in two groups. Some authors aimed at optimal instruction identification, developing algorithms with exponential worst-case complexity. Other works reduced 

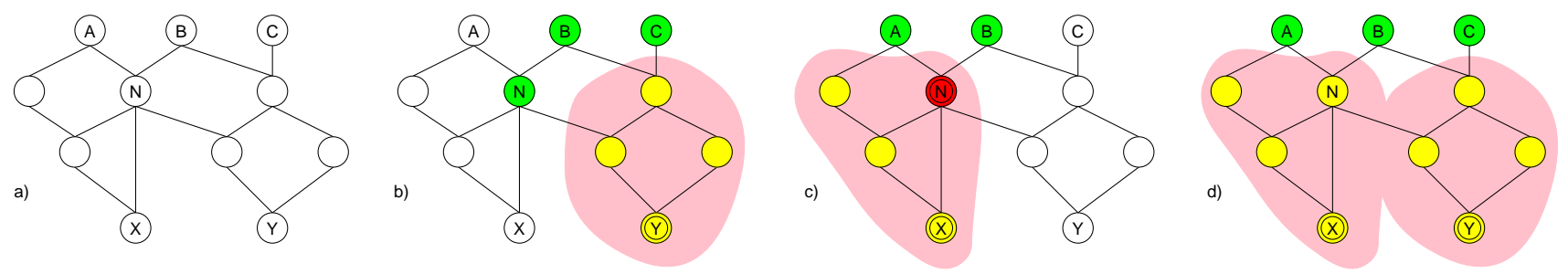

Figure 1. a) A data-flow graph (all edges are towards the bottom of the figure); b) a valid 1-output convex cut; nodes N, B, C are inputs to the output node Y; c) an invalid 1-output convex cut, where $\mathrm{N}$ appears as an additional output besides $\mathrm{X}$; $\mathrm{d}$ ) a valid 2-output convex cut.

the complexity, at the expense of optimality or generality.

Atasu et al. developed a widely known optimal algorithm [3], which Pozzi et al. further refined with additional pruning techniques [12]. Both algorithms are based on subgraph enumeration: they exhaustively explore the search space algorithm, pruning it through constraint propagation. Neither poses limits on the latency or connectedness of the instructions. The latter algorithm is reasonably efficient even for large basic blocks, but its performance quickly deteriorates if the custom instructions can have multiple outputs. An Integer Linear Programming formulation of the same problem was presented in [2]: in this case, the enumeration of subgraphs is implicit in the formulation's constraints, and the worst-case complexity is still exponential.

Other authors simplify the problem formulation and then find exact solutions for it. For example, Choi et al. [6] limit the latency (depth) of subgraphs, and Yu and Mitra [13] only focus on identifying connected custom instructions. Our algorithm can be adapted to run faster under this kind of limitation.

Other approaches include greedy techniques such as those in Clark et al. [8] and Biswas et al. [4]. While fast, these algorithms have limited effectiveness [7]. More recent work seems to focus on optimal algorithms, possibly using subgraph enumeration as a building block.

The algorithm we present is based on multiple-vertex dominators. Also known as generalized dominators, they were introduced by Gupta in [10]. Single-vertex dominators are a widely studied problem [1], but they are rare in circuit graphs or data-flow graphs: multiple-vertex dominators are more common and can be useful to explore or simplify such graphs. Gupta presents an algorithm to enumerate all multiple-vertex dominators, but only with exponential worst-case complexity. A smaller bound for this problem was never published, and it is an open problem to find an efficient way of representing the set of all possible dominators.

Dubrova et al. [9], however, do present an algorithm to enumerate $k$-vertex dominators in polynomial time $O\left(n^{k}\right)$. As we show in section 5, we can employ this algorithm successfully to find optimal custom instructions.
The authors report that the algorithm is very slow for $k>2$; however, we developed pruning techniques for subgraph enumeration, that make this algorithm practical also for somewhat higher values of $k$, and for graphs with a thousand nodes or more.

\section{Problem statement}

The data flow of each basic block is represented by a graph $G(V, E)$. The graph $G$ may have an arbitrary number of root vertices $I_{\text {ext }}$, that is vertices that have no predecessors. These vertices represent input variables of the basic block. The graph may also have a set of vertices $O_{e x t}$ that is a superset of those vertices that have no successors. Figure 1(a) shows an example data-flow graph with 3 roots (vertices A, B, C) and $2 O_{\text {ext }}$ vertices (X and $\mathrm{Y}$ ).

A cut is defined on a rooted, direct, acyclic graph. $G$ is transformed into a rooted graph, by augmenting it with a single vertex that is a predecessor of every vertex in $I_{\text {ext }}$. We also create an additional vertex (the sink) and connect $O_{e x t}$ to the sink. This way, the reverse graph of $G$ also a rooted graph, which is useful when computing postdominators. The definition of cut, and in particular of convex cut, are as follows.

Definition 1 (Cut): A cut $S$ is a subgraph of a graph $G$. We call inputs of $S$ the set I $(S)$ of predecessor vertices of those edges which enter the cut $S$ from the rest of the graph $G$, that is $\mathrm{I}(S)=\bigcup_{v \in S} \operatorname{pred}(v) \backslash S$. Similarly, we call outputs of $S$ the set $\mathrm{O}(S)$ of vertices which are part of $S$, but have at least one successor $v \notin S$.

Definition 2 (Convex cut): A cut $S$ is convex if there is no path from a vertex $u \in S$ to another vertex $v \in S$ which contains a vertex $w \notin S$.

The shaded areas in figure 1(b)(c)(d) are all examples of a convex cut. Nodes with a double border are outputs ${ }^{1}$ and grey nodes are inputs.

The microarchitecture may pose several additional constraints on the cuts that can be considered valid. First of all,

\footnotetext{
${ }^{1}$ The terms vertex and node will be used interchangeably.
} 
the values $N_{\text {in }}$ and $N_{\text {out }}$ indicate the maximum number of read and write ports in the register file, respectively, which a custom instruction can use. Secondly, some nodes of $G$ may be forbidden, that is, they may not be included in a cut ${ }^{2}$.

Some forbidden nodes will be marked as such by the user, and represent operations that are not allowed in a special instruction - for example, loads and stores if the custom functional unit cannot have any memory port. In addition to these nodes, the algorithm will consider other nodes to be forbidden. $I_{\text {ext }}$ nodes are implicitly forbidden, because their values are calculated outside the basic block. Likewise, the newly introduced source and sink will be considered forbidden because they do not map to an actual computation in the program. We will denote forbidden nodes with $F$.

Thus, given a graph $G$, the posed problem is to find all convex cuts $S \subseteq G$ under the constraints that $|\mathrm{I}(S)| \leq N_{\text {in }}$, $|\mathrm{O}(S)| \leq N_{\text {out }}$, and $S \cap F=\emptyset$.

In the remainder of this paper, we add another condition for the validity of the convex cut. For each input $w \in \mathrm{I}(S)$, there is a vertex $v \in S$, such that at least one path from the root of $G$ to vertex $v$ contains $w$ but not any other input of $S$.

This condition excludes from consideration a few valid cuts, namely those where an input $w$ only has other inputs as predecessors (in this case, the inputs to $w$ must be predecessors of $w$ for the cut to be convex). These cuts violate the condition we just added because all the paths from the root of $G$ to $w$ contain other inputs. Given the cut in figure 1(d), we would have such a cut if the node marked as $\mathrm{N}$ was a fourth input. All paths from the root to $\mathrm{N}$ would pass through the inputs A and B.

Note that all the predecessors of $w$ need to be inputs, or there would be a path from the root of $G$ to $v$ that only contains the input $w$. Then, all the inputs but $w$ will be inputs to the valid cut $S \cup\{w\}$. This cut-like the one in figure 1(d) - will be found by our algorithm, and can be used to find the cuts that were lost by the addition of the technical condition.

\section{Properties of convex cuts}

We can define more characteristics and prove some properties of convex cuts.

Definition 3 (Inputs to a vertex): In a convex cut $S$, the inputs to a vertex $v$ are defined to be the set of vertices $I_{v}(S) \subseteq \mathrm{I}(S)$ such that 1) all paths from the root of $G$ to $v$ contains at least one vertex $i \in I_{v}(S)$;2) for every vertex $i \in I_{v}(S)$, at least one path from the root to $v$ contains $i$.

For example, $A$ and $B$ are the inputs to $X$ in figure 1(d). There is an important link between the generalized dominators of an output $o$, and the inputs to $o$. Generalized dominators are defined as follows on a rooted graph $G$.

\footnotetext{
${ }^{2}$ Note that forbidden nodes may still be chosen as inputs to a cut.
}

Definition 4 (Generalized dominator): A set of vertices $V$ in a rooted graph $G$ dominates a vertex $v$ iff it meets the following two conditions: 1) all paths from the root of $G$ to vertex $v$ contain at least one vertex $w \in V ; 2$ ) for each vertex $w \in V$, there is at least one path from the root of $G$ to vertex $v$, which contains $w$ but not any other vertex in $V$.

We can then prove the following theorem and provide the link between inputs and generalized dominators:

Theorem 1: If $S \subseteq G$ identifies a convex cut, then for every output $o$ of $S$ the set of vertices $I_{o}(S)$ that are inputs to $o$ is a generalized dominator of $o$ in $G$.

Proof. From definition 3, every path from the root to $o$ contains at least one of the vertices in $I_{o}(S)$. Condition 1 is then verified in the definition of generalized dominators.

With the more restrictive definition of convex cut that we presented above, they also satisfy condition 2. Because of that restriction, for each input $i \in I_{o}(S)$, there is a vertex $v \in S$, such that at least one path from the root of $G$ to vertex $v$ contains $i$ but not any other input of $S$.

In particular, we can find one such path for any $v$ that is a successor of $i$ and not an input. If we pick a vertex that is also contained in a path between $i$ and $o$ (there will always be such a path, because $i$ is an input to $o$ ), then the path can be extended to stop at $o$ instead of $v$. This proves that condition 2 is also verified.

For example, given the convex cut in figure 1(b), this theorem proves that the set of inputs to the cut is a generalized dominator of the output. The theorem provides a useful starting point to derive the nodes in a convex cut from its input and output vertices, and to prove that inputs and outputs uniquely identify the convex cut. However, this proof needs another definition:

Definition 5 (Vertices between $V$ and $w): B(V, w)$, the set of vertices between a set $V$ and a vertex $w$, is the set of vertices contained by at least one path between a vertex $v \in V$ and $w$ (if there is such a path). The starting vertex of the path is not included, while the final vertex $w$ is.

For example, in figure 1(b), the shaded area represents the vertices between its inputs (nodes $\mathrm{B}, \mathrm{C}$ and $\mathrm{N}$ ), and the output node Y. Note that $B(V, w)$ can be computed easily; the complexity is linear in the size of the set $B$ itself, and hence $O(n)$.

Theorem 2: Any convex cut is uniquely identified by its sets of input and output vertices, respectively $\mathrm{I}(S)$ and $\mathrm{O}(S)$. In other words, two convex cuts of the same graph are equal iff they have the same inputs and outputs.

Proof. If two convex cuts $S$ and $T$ are equal, they have the same sets of inputs and outputs. This is true because the inputs and outputs of a convex cut are functions of the vertices in the cut. 
If two convex cuts have the same sets of inputs and outputs, they are equal. To prove this part, we consider the cut $S^{\prime}=\bigcup_{v \in \mathrm{O}(S)} B(\mathrm{I}(S), v) \backslash \mathrm{I}(S)$, and prove that $S=S^{\prime}$.

If a vertex $v$ was in $S^{\prime}$ but not in $S$, then there would be a path from an input to an output going through $v \notin$ $S$, violating the definition of convexity. This because $S^{\prime}$ is defined to include all the vertices, along every possible path from an input to an output.

If a vertex $v$ was in $S$ but not in $S^{\prime}$, this means that no path from the inputs to the outputs contains $v$. However, every path from the root to $v$ must contain at least one input in $I_{v}(S)$. Then, there is always a path from this input to $v$-that is, $v \in B(\mathrm{I}(S), v)$. If $v$ was an output, $B(\mathrm{I}(S), v)$ would be included in $S^{\prime}$, hence $v \in S^{\prime}$ and we have a contradiction. If $v$ was not an output, there must be a path from $v$ to an output $o$, otherwise at least one successor of $v$ would not belong in $S$ and $v$ would be an output. Therefore $v \in B(\mathrm{I}(S), o)$ and we also have a contradiction.

We proved that a convex cut is uniquely identified by the sets of inputs and outputs. However, the reverse is not true: given two sets of vertices $I$ and $O$, they identify a convex cut only under rather strict conditions. The following weaker theorem, nevertheless, forms the basis, and at the same time the correctness proof, for our algorithm.

Theorem 3: Given two sets of vertices $I$ and $O$, if for every vertex $o_{j} \in O$, there is a set of vertices $I_{j} \subseteq I$ such that $I_{j}$ dominates $o$, then $S=\bigcup_{o_{j} \in O} B\left(I_{j}, o_{j}\right) \backslash I_{j}$ is a convex cut with $\mathrm{I}(S) \subseteq I$.

Proof. The convexity of $S$ derives directly from the definition of $B\left(I_{j}, o_{j}\right)$ : all the vertices on a path between an input and an output are included in $S$, therefore no such path can cross a vertex that is not part of $S$.

If an $i \in \mathrm{I}(S)$ was not in any $I_{j}$, we would have a path from the root to every $o_{j}$ that passed through a vertex not in $I_{j}$. This contradicts the hypothesis that $I_{j}$ dominates $o_{j}$.

This theorem provides a way to compute the vertices in a cut in $O(n)$ time (since $B\left(I_{j}, o_{j}\right)$ can also be computed in linear time). It also guarantees that the cut will not have any more inputs. On the other hand, it does not guarantee that the cut will have precisely the requested inputs and outputs-see figure 1(c) for an example where an additional output appears between B and X. As we will see later, however, this can actually be exploited to speed up our algorithm.

\section{Algorithm}

Since the convex cuts in $G$ are uniquely identified by its input and output vertices, it is possible to enumerate them by coupling every possible set of outputs with all the possible sets of inputs. If, as in the posed problem, we put a constraint on the number of inputs and outputs, the number

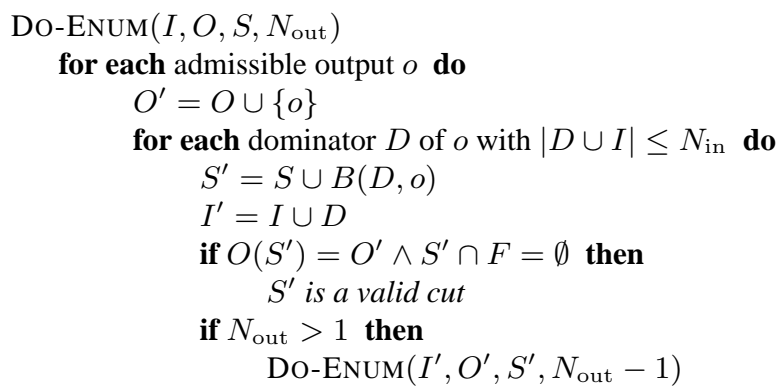

PoLY-ENum()

Do-Enum $\left(\emptyset, \emptyset, \emptyset, N_{\text {out }}\right)$

\section{Figure 2. A polynomial-time algorithm for subgraph enumeration}

of valid convex cuts is clearly polynomial; more precisely, it is $O\left(n^{N_{\text {in }}+N_{\text {out }}}\right)$.

Section 5.1 describes a basic solution to the problem, which would be feasible only for small basic blocks. By switching to an incremental mode of operation, the choice of inputs and outputs can be pruned at each step: section 5.2 details the refinements in the algorithm, and section 5.3 outlines the corresponding pruning techniques.

\subsection{Basic solution}

An implementation of the algorithm is presented in figure 2. To find all sets of vertices that can be inputs to an output vertex $o$, we try all the (possibly multiple-vertex) dominators of the output. We start by picking an output node, and explore all its dominators. After finding one, we may add another output node and recursively explore the new output's dominators.

In principle, any $n$-uple of vertices could be taken as an output. In practice, not all of them are useful: an output vertex $o_{2}$ is not admissible if another output vertex $o_{1}$ postdominates it. Note that a vertex $v \in O_{\text {ext }}$ will not be postdominated by any vertex but the artificial sink, because it is connected by an edge to the sink.

Note how, in agreement with theorem 3, we need an additional check that the cut really has no outputs besides $O$. If a cut fails this test, as in figure 1(c), it may still be extended with a new output and become valid: for example, node $\mathrm{N}$ itself may be added, or node $\mathrm{Y}$ may be added yielding the cut in figure $1(\mathrm{~d})$.

To analyze the complexity of the algorithm we take into account each step. Setting up the algorithm includes the computation of single- and multiple-vertex dominators (with maximum cardinality $N_{\text {in }}$ ) of every node. Let $O(\tau(n))$ be the complexity of computing single-vertex dominators on a graph with $n$ vertices. In theory, this can be as low as $O(n)$ but, as mentioned in section 2, practically used algorithms have a slightly higher complexity. Computing single-vertex dominators (and postdominators) requires 
a time $O(\tau(n))$, while computing the multiple-vertex dominators requires a time $O\left(n^{N_{\text {in }}-1} \tau(n)\right)$.

Given the inputs and outputs of a cut, the nodes that are part of the cut can be enumerated in $O(n)$ time. Checking that the cut has no extraneous outputs has the same cost. Function POLY-ENUM has a complexity of $O\left(n^{N_{\text {in }}+N_{\text {out }}+1}\right)$. As this dominates all the setup phases, it is also the complexity of subgraph enumeration for the algorithm in figure 2 .

\subsection{Incremental operation}

The pseudo-code in figure 2 is agnostic of the algorithm used to compute multiple-vertex dominators. Since we know of only one such algorithm with polynomial complexity, we can tailor our implementation to it in order to improve its speed.

The multiple-vertex dominator algorithm from [9] picks every possible seed set $\left\{v_{1}, \ldots, v_{n-1}\right\}$. Then, it removes the vertices in the seed set from the graph, together with any other node they dominate. Then, if a node $u$ dominates a node $w$ in this reduced graph, $\left\{v_{1}, \ldots, v_{n-1}, u\right\}$ is a multiple-vertex dominator of $w$ in the original graph.

The exploration of the seed sets (which covers the inner for each loop of figure 2) can be done recursively, just like for the output nodes. Every recursive call pushes a vertex on the seed set, calls the Lengauer-Tarjan algorithm [11] on the reduced graph, and then pops the vertex. There will be up to $N_{\text {in }}-1$ recursive calls, giving the algorithm in figure 3.

Note how $S=\bigcup_{o \in O} B(D, o)$ is built incrementally. Pushing an input or an output adds nodes to $S$, such that on every recursive call $S$ can only grow. In particular, the newly added nodes for an input $v$ are $S_{\text {new }}=B(\{v\}, o) \backslash S$. For an output $o$, they are $S_{\text {new }}=B(I, o) \backslash S$. As a further optimization, our implementation maintains a single copy of $S$. We keep track of when each node was added to $S$, and remove them before leaving the invocation that added them.

Unlike the naïve algorithm, this algorithm does everything in a single pass without any setup phase. The complexity is the same as for the previously presented one, that is $O\left(n^{N_{\text {in }}+N_{\text {out }}+1}\right)$. In the for each loops, the cost of invoking PICK-INPUTS dominates the linear-time work to update $S$; when CHECK-CUT is called, instead, updating $S$ is covered by the additional +1 in the exponent.

\subsection{Pruning techniques}

The algorithms we presented have a high asymptotic complexity. The incremental algorithm however enables pruning techniques that enhance performance by reducing the $n$ in the complexity. These make the algorithm practical even for graphs with 1,000 or more nodes ${ }^{3}$.

\footnotetext{
${ }^{3}$ These techniques are only explaned briefly in this paper; an extended description is available in [14].
}

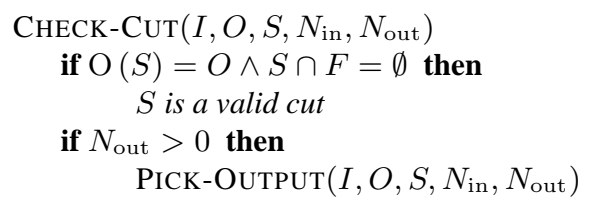

Pick-Inputs $\left(I, o, O, S, N_{\text {in }}, N_{\text {out }}\right)$

$\triangleright$ the next line invokes Dubrova et al.'s algorithm

for each node $w$ such that $I \cup\{w\}$ dominates $O$ do

$I^{\prime}=I \cup\{w\}$

$S^{\prime}=S \cup B(\{w\}, o)$

CHECK-Cut $\left(I^{\prime}, O, S^{\prime}, N_{\text {in }}-1, N_{\text {out }}\right)$

if $N_{\mathrm{in}}>1$ then

$\triangleright$ add a node to the seed set

for each ancestor $i$ of $o$ do

$$
\begin{aligned}
& I^{\prime}=I \cup\{i\} \\
& S^{\prime}=S \cup B(\{i\}, o)
\end{aligned}
$$$$
\text { PiCK-Inputs }\left(I^{\prime}, o, O, S^{\prime}, N_{\text {in }}-1, N_{\text {out }}\right)
$$

Pick-Output $\left(I, O, S, N_{\text {in }}, N_{\text {out }}\right)$

for each admissible output $o$ do

$$
O^{\prime}=O \cup\{o\}
$$$$
S^{\prime}=S \cup B(I, o)
$$

if $I$ dominates $O$ then

$$
\text { CheCK-Cut }\left(I, O^{\prime}, S^{\prime}, N_{\text {in }}, N_{\text {out }}-1\right)
$$

elseif $N_{\text {in }}>0$ then

$\operatorname{Pick}-\operatorname{InpUts}\left(I, o, O^{\prime}, S^{\prime}, N_{\mathrm{in}}, N_{\text {out }}-1\right)$

POLY-ENUM-INCR()

Pick-Output $\left(\emptyset, \emptyset, \emptyset, N_{\text {in }}, N_{\text {out }}\right)$

\section{Figure 3. Building $S$ incrementally}

First of all, some cuts can be determined to be invalid just by looking at $S$. This is the primary added value of the incremental algorithm described in section 5.2. Cuts that include a forbidden node, for example, can be discarded right away, as the problem statement forbids them.

Second, we can treat cuts that have other outputs than $O$ as acceptable, as long as the total number of outputs does not exceed $N_{\text {out }}$. For example, the cut in figure 1(c) may be accepted as a 2-output subgraph.

Finally, forbidden nodes are effectively partitioning the search space. When a node $v$ is picked as an output, if a forbidden node $w$ is an ancestor of $v, w$ 's ancestors will not be valid inputs to $v$.

\section{Results}

In order to evaluate our algorithm's performance, we collected the data-flow graphs of 250 basic blocks from MiBench. The sizes of the blocks range from 10 to 1196 nodes. We also used four synthetic data-flow graphs, treeshaped as in figure 4 . Their depth varies from 4 to 7 levels. We found them empirically to exhibit worst-case performance for algorithms like [3] and [12]; in particular, for [3] the complexity can be proved to be exponential, $O\left(1.6^{n}\right)$, on this kind of graph. 


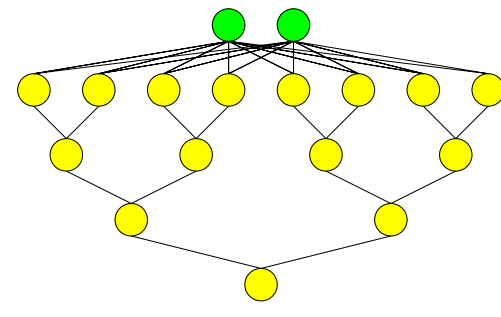

Figure 4. A tree-shaped data-flow graph, the worst case for algorithms such as [12].

The graph in figure 5 compares, for each basic block, the speed of our algorithm versus the implementation in [12]. Subgraphs are enumerated with a constraint of four inputs and two outputs. For each basic block processed, a point is plotted at the intersection between our algorithm's execution time (on the $\mathrm{X}$ axis) and that of [12] (on the $\mathrm{Y}$ axis). Therefore, data points above the diagonal line represent executions where our algorithm is faster, and data points below the straight line represent those cases where our algorithm is slower. Different symbols group the data points in four clusters; three of these correspond to different sizes and the fourth is reserved to the synthetic DFGs, shaped as in figure 4.

The algorithm's performance is in general better than that of [12]. Some exceptions are expected, because both algorithms employ pruning techniques whose effectiveness can vary widely for different data-flow graph topologies. [12] also seems to achieve a similar polynomial time bound; however, while already stated in [12], this has not been proved formally so far.

In fact, the main contribution of this paper is the proof that the enumeration problem actually has polynomial complexity in $n$. State-of-the-art algorithms explored a binary search space, where each node could either be part of the subgraph or not. We look at the search space from a different perspective: each convex subgraph can have at most $N_{\text {in }}$ inputs and $N_{\text {out }}$ outputs nodes, and these nodes univocally identify the subgraph that lays between them.

Finally, we show here only the performance of the enumeration algorithm presented, and we are not concerned with the speedup enabled by Instruction Set Extensions designed using this technique: the effectiveness of using this technique for identification of custom instruction has been widely validated by past papers, including $[12,3,7]$.

\section{Conclusion}

We presented a novel algorithm for full enumeration of the subgraphs of a given data-flow graph. The algorithm supports arbitrary input/output constraints, is not restricted to connected subgraphs and, to our knowledge, is the first one to be presented with polynomial time complexity.

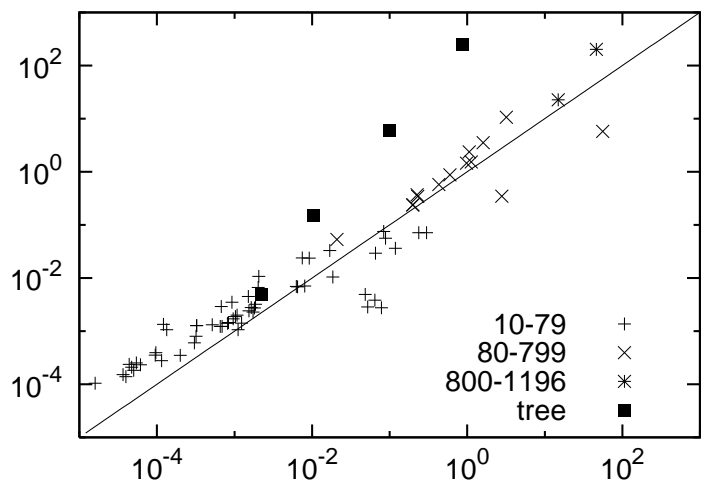

Figure 5. Run time comparison with [12].

This algorithm was successfully used in in our compiler toolchain [5]; full subgraph enumeration allows detection of high-performance custom instruction sets, yielding speedups up to $6 \mathrm{x}$. In addition to having a known polynomial bound to the complexity, the performance of the algorithm is in parallel, and usually better, than the algorithm in [12]. This represents a further contribution to the field of automatic identification of Instruction Set Extensions for embedded processors.

\section{References}

[1] A. V. Aho and J. D. Ullman. Theory of Parsing, Translation and Compiling. Prentice Hall, 1973.

[2] K. Atasu, G. Dündar, and C. Özturan. An integer linear programming approach for identifying instruction-set extensions. In Proc. of CODES+ISSS, 2005.

[3] K. Atasu, L. Pozzi, and P. Ienne. Automatic application-specific instruction-set extensions under microarchitectural constraints. In Proc. of DAC, 2003.

[4] P. Biswas, S. Banerjee, N. Dutt, L. Pozzi, and P. Ienne. ISEGEN: Generation of high-quality instruction set extensions by iterative improvement. In Proc. of DATE, 2005.

[5] P. Bonzini and L. Pozzi. Code transformation strategies for extensible embedded processors. In Proc. of CASES, 2006.

[6] H. Choi, J.-S. Kim, C.-W. Yoon, I.-C. Park, S. H. Hwang, and C.-M. Kyung. Synthesis of application specific instructions for embedded DSP software. In IEEE TC, June 1999.

[7] N. Clark, A. Hormati, and S. Mahlke. Scalable subgraph mapping for acyclic computation accelerators. In Proc. of CASES, 2006.

[8] N. Clark, H. Zhong, and S. Mahlke. Processor acceleration through automated instruction set customisation. In Proc. of MICRO, 2003.

[9] E. Dubrova, M. Teslenko, and A. Martinelli. On relation between non-disjoint decomposition and multiple-vertex dominators. In Proc. of ISCAS, 2004.

[10] R. Gupta. Generalized dominators and post-dominators. In Proc. of POPL, 1992.

[11] T. Lengauer and R. E. Tarjan. A fast algorithm for finding dominators in a flowgraph. In ACM TOPLAS, 1979.

[12] L. Pozzi, K. Atasu, and P. Ienne. Optimal and approximate algorithms for the extension of embedded processor instruction sets. In IEEE TCAD, July 2006.

[13] P. Yu and T. Mitra. Scalable custom instructions identification for instruction set extensible processors. In Proc. of CASES, 2004.

[14] P. Bonzini and L. Pozzi Polynomial-time subgraph enumeration for automated instruction set extension. TR 2006/07, University of Lugano, 2006. http: //www.inf.unisi.ch/file/pub/ 15/bonzini-pozzi-2006-07.pdf 